\title{
Multiplicity and degree as bi-Lipschitz invariants for complex sets
}

\author{
Javier Fernández de Bobadilla, Alexandre Fernandes and J. Edson Sampaio
}

\begin{abstract}
We study invariance of multiplicity of complex analytic germs and degree of complex affine sets under outer bi-Lipschitz transformations (outer bi-Lipschitz homeomorphims of germs in the first case and outer bi-Lipschitz homeomorphims at infinity in the second case). We prove that invariance of multiplicity in the local case is equivalent to invariance of degree in the global case. We prove invariance for curves and surfaces. In the way we prove invariance of the tangent cone and relative multiplicities at infinity under outer bi-Lipschitz homeomorphims at infinity, and that the abstract topology of a homogeneous surface germ determines its multiplicity.
\end{abstract}

\section{Introduction}

We study invariance of multiplicity of complex analytic germs and degree of complex affine sets under outer bi-Lipschitz transformations: outer bi-Lipschitz homeomorphisms of germs in the first case and outer bi-Lipschitz homeomorphisms at infinity in the second case (see Definition 33.

The local problem may be seen as a bi-Lipschitz version of Zariski multiplicity problem 16, that asks whether the embedded topology of complex hypersurface germs determine their multiplicity. It is well known that the abstract topological type of hypersurface germs (i.e the topology of the intersection of the germs with a small ball) does not determine the multiplicity. This is clear for curves, since their abstract topology is too simple, but also in the case of surfaces, where the abstract topology is very rich, examples are known (see Example 2.22 at 9 ).

In contrast with this situation we conjecture, as we will make precise shortly, that the local and at infinity outer bi-Lipschitz geometry (which does not take into account the embedding) determine the multiplicity and degree of complex sets respectively. It is known that the inner bi-Lipschitz geometry does not determine multiplicity (for example complex curves).

Conjecture $\tilde{\mathbf{A}} \mathbf{1}(d)$ Let $X \subset \mathbb{C}^{n}$ and $Y \subset \mathbb{C}^{m}$ be two complex analytic sets with $\operatorname{dim} X=$ $\operatorname{dim} Y=d, 0 \in X$ and $0 \in Y$. If their germs at $0 \in \mathbb{C}^{n}$ and $0 \in \mathbb{C}^{m}$, respectively, are outer biLipschitz homeomorphic, i.e. there exists an outer bi-Lipschitz homeomorphism $\varphi:(X, 0) \rightarrow$ $(Y, 0)$, then their multiplicities $m(X, 0)$ and $m(Y, 0)$ are equal.

Conjecture $\mathbf{A} \mathbf{1}(d)$ Let $X \subset \mathbb{C}^{n}$ and $Y \subset \mathbb{C}^{m}$ be two complex algebraic sets with $\operatorname{dim} X=$ $\operatorname{dim} Y=d$. If $X$ and $Y$ are outer bi-Lipschitz homeomorphic at infinity (see Definition 3), then we have the equality $\operatorname{deg}(X)=\operatorname{deg}(Y)$.

\footnotetext{
2000 Mathematics Subject Classification 14B05 (primary), 32S50 (secondary).

The first named author is partially supported by IAS and by ERCEA 615655 NMST Consolidator Grant, MINECO by the project reference MTM2013-45710-C2-2-P, by the Basque Government through the BERC 2014-2017 program, by Spanish Ministry of Economy and Competitiveness MINECO: BCAM Severo Ochoa excellence accreditation SEV-2013-0323, by Bolsa Pesquisador Visitante Especial (PVE) - Ciencias sem Fronteiras/CNPq Project number: 401947/2013-0 and by Spanish MICINN project MTM2013-45710-C2-2-P. The second named author was partially supported by CNPq-Brazil grant 302764/2014-7.

The third named author was partially supported by the ERCEA 615655 NMST Consolidator Grant and also by the Basque Government through the BERC 2014-2017 program and by Spanish Ministry of Economy and Competitiveness MINECO: BCAM Severo Ochoa excellence accreditation SEV-2013-0323.
} 
Conjecture Â1 $(d)$ was approached by some authors. G. Comte 3 proved that the multiplicity of complex analytic germs is invariant under outer bi-Lipschitz homeomorphism with Lipschitz constants close enough to 1 (this is a severe assumption). Neumann and Pichon in [10], with previous contributions of Pham and Teissier in $[\mathbf{1 2}$ and Fernandes in $[\mathbf{4}$, proved that the outer bi-Lipschitz geometry of plane curves determines the Puiseux pairs, and as a consequence proved Â1(1). More recently, W. Neumann and A. Pichon in 11 showed that the multiplicity is an outer bi-Lipschitz invariant in the case of normal surface singularities, as a consequence of a very detailed and involved study of the outer bi-Lipschitz geometry for that class. In the non-normal surface case the only partial contribution is the fact that the embedded bi-Lipschitz geometry determines multiplicity in the hypersurface case (see [5]). Conjecture A1 $(d)$ is largely unexplored up to our knowledge.

Our main results are the following. We prove that Conjecture $\tilde{A} 1(d)$ is equivalent to Conjecture $\mathrm{A} 1(d)$ (Theorem 3.3). We prove the conjectures for curves and surfaces $(d=1,2)$ (Corollary 3.2 and Theorem 3.4). For general $d$ we prove in Theorem 3.6 that Conjecture A1 $(d)$ holds for algebraic hypersurfaces in $\mathbb{C}^{n}$ whose all irreducible components of their tangent cones at infinity have singular locus with dimension $\leq 1$ and, as an immediate corollary of it, we obtain that degree of complex algebraic surfaces in $\mathbb{C}^{3}$ is an embedded bi-Lipschitz invariant at infinity.

The way to reach these results is to prove that the outer bi-Lipschitz geometry at infinity determines the tangent cone at infinity and the relative multiplicities at infinity (Theorem 3.1. These are versions "at infinity" of the corresponding results for germs in $\mathbf{1 3}$ and $\mathbf{5}$ respectively. In order to have an idea of the ingredients of the statement let us remark that in the hypersurface case the tangent cone at infinity is the set defined by the highest degree form of the defining equation. The relative multiplicities at infinity are the exponents appearing in the factorization in irreducible components of the highest degree form. Precise definitions are in Section 2 ,

The case $d=1$ comes very easily from the last mentioned result. For the $d=2$ case the new idea is to find the degree of a homogeneous irreducible affine algebraic set $S$ as the torsion part of a cohomology group of $S \backslash\{0\}$. We use the Leray spectral sequence associated with its projectivization for that purpose. In particular we show that the abstract topology of a homogeneous surface germ determines its multiplicity.

The organization of the paper is as follows. In Section 2 we recall the necessary basic definitions and introduce relative multiplicities at infinity. In Section 3 we prove the results described above.

REMARK 1. After this paper was submitted the last two authors, together with Birbrair and Verbitsky, proved that conjectures $\tilde{A} 1(d)$ and $A 1(d)$ are false for $d \geq 3$, by showing explicit counter-examples (see $\mathbf{1}$ ). So our arguments cannot be generalized further to work in higher dimension.

\section{Preliminaries}

\subsection{Multiplicity, degree and tangent cones}

Definition 1. Let $A$ be a closed algebraic subset in $\mathbb{C}^{n}$. We define the degree of $A$ to be the degree of its projective completion $[2$. 
REMARK 2. If $A$ is a homogeneous algebraic set in $\mathbb{C}^{n}$ (i.e defined by homogeneous polynomials), its degree coincides with its multiplicity at the origin of $\mathbb{C}^{n}$ (see 2 for a definition of multiplicity for complex analytic germs in $\mathbb{C}^{n}$ ).

Now we set the definition of tangent cone at infinity that we will use along the paper and we list some of its properties.

Definition 2. Let $A \subset \mathbb{R}^{n}$ be an unbounded subset. We say that $v \in \mathbb{R}^{n}$ is a tangent vector of $A$ at infinity if there is a sequence of points $\left\{x_{i}\right\}_{i \in \mathbb{N}} \subset A$ such that $\lim _{i \rightarrow \infty}\left\|x_{i}\right\|=+\infty$ and there is a sequence of positive numbers $\left\{t_{i}\right\}_{i \in \mathbb{N}} \subset \mathbb{R}^{+}$such that

$$
\lim _{i \rightarrow \infty} \frac{1}{t_{i}} x_{i}=v
$$

Let $C_{\infty}(A)$ denote the set of all tangent vectors of $A$ at infinity. This subset $C_{\infty}(A) \subset \mathbb{R}^{n}$ is called the tangent cone of $A$ at infinity.

Proposition 2.1 Proposition 4.4 in $\left[\mathbf{6}\right.$. Let $Z \subset \mathbb{R}^{n}$ be an unbounded semialgebraic set. $A$ vector $v \in \mathbb{R}^{n}$ belongs to $C_{\infty}(Z)$ if, and only if, there exists a continuous semialgebraic curve $\gamma:(\varepsilon,+\infty) \rightarrow Z$ such that $\lim _{t \rightarrow+\infty}|\gamma(t)|=+\infty$ and $\gamma(t)=t v+o_{\infty}(t)$, where $g(t)=o_{\infty}(t)$ means $\lim _{t \rightarrow+\infty} \frac{g(t)}{t}=0$.

Let $X \subset \mathbb{C}^{n}$ be a complex algebraic subset. Let $\mathcal{I}(X)$ be the ideal of $\mathbb{C}\left[x_{1}, \cdots, x_{n}\right]$ given by the polynomials which vanish on $X$. For each $f \in \mathbb{C}\left[x_{1}, \cdots, x_{n}\right]$, let us denote by $f^{*}$ the maximum degree form of $f$. Define $\mathcal{I}^{*}(X)$ to be generated by the $f^{*}$ when $f \in \mathcal{I}(X)$.

Proposition 2.2 Theorem 1.1 in [8. Let $X \subset \mathbb{C}^{n}$ be a complex algebraic subset. Then, $C_{\infty}(X)$ is the affine algebraic subset defined by $\mathcal{I}^{*}(X)$. We emphasize that we take $C_{\infty}(X)$ as a set, with reduced structure.

Among other things, this result above says that tangent cones at infinity of complex algebraic sets in $\mathbb{C}^{n}$ are complex algebraic subsets as well.

\subsection{Outer bi-Lipschitz homeomorphism at infinity}

All the Euclidean subsets are equipped with the induced Euclidean distance (outer metric). So, all the Lipschitz mappings mentioned here are supposed to be Lipschitz with respect to the outer metric, this is why they are called outer Lipschitz or bi-Lipschitz mappings

Definition 3. Let $X \subset \mathbb{R}^{n}$ and $Y \subset \mathbb{R}^{m}$ be two subsets. We say that $X$ and $Y$ are outer bi-Lipschitz homeomorphic at infinity, if there exist compact subsets $K \subset \mathbb{R}^{n}$ and $\widetilde{K} \subset$ $\mathbb{R}^{m}$ and an outer bi-Lipschitz homeomorphism $\phi: X \backslash K \rightarrow Y \backslash \widetilde{K}$.

We finish this subsection reminding the invariance of the tangent cone at infinity under outer bi-Lipschitz homeomorphisms at infinity. 
Proposition 2.3 Theorem 4.5 in $\left[\mathbf{6}\right.$. Let $X \subset \mathbb{R}^{n}$ and $Y \subset \mathbb{R}^{m}$ be unbounded semialgebraic subsets. If $X$ and $Y$ are outer bi-Lipschitz homeomorphic at infinity, then there is an outer bi-Lipschitz homeomorphism $d \varphi: C_{\infty}(X) \rightarrow C_{\infty}(Y)$ with $d \varphi(0)=0$.

\subsection{Relative multiplicities at infinity}

Let $X \subset \mathbb{C}^{n}$ be a complex algebraic set with $p=\operatorname{dim} X \geq 1$. Let $X_{1}, \cdots, X_{r}$ be the irreducible components of $C_{\infty}(X)$.

Below we define relative multiplicities at infinity.

Let $\pi: \mathbb{C}^{n} \rightarrow \mathbb{C}^{p}$ be a linear projection such that

$$
\pi^{-1}(0) \cap\left(C_{\infty}(X)\right)=\{0\} .
$$

Therefore, $\left.\pi\right|_{X}: X \rightarrow \mathbb{C}^{p}$ (resp. $\left.\pi\right|_{C_{\infty}(X)}: C_{\infty}(X) \rightarrow \mathbb{C}^{p}$ ) is a ramified cover with degree equal to $\operatorname{deg}(X)\left(\right.$ resp. $\left.\operatorname{deg}\left(C_{\infty}(X)\right)\right)$ (see $[2]$, Corollary 1 in the page 126). In particular, $\left.\pi\right|_{X_{j}}: X_{j} \rightarrow \mathbb{C}^{p}$ is a ramified cover with degree equal to $\operatorname{deg}\left(X_{j}\right)$, for each $j=1, \cdots, r$. Moreover, if the ramification locus of $\left.\pi\right|_{X}$ (resp. $\left.\left.\pi\right|_{C_{\infty}(X)}\right)$ is not empty, it is a codimension 1 complex algebraic subset $\sigma(X)$ (resp. $\left.\sigma\left(C_{\infty}(X)\right)\right)$ of $\mathbb{C}^{p}$. Let us denote $\Sigma_{X}=\left(\left.\pi\right|_{X}\right)^{-1}(\sigma(X))$ and $\Sigma_{X}^{\prime}=\left(\left.\pi\right|_{C_{\infty}(X)}\right)^{-1}\left(\sigma\left(C_{\infty}(X)\right)\right)$.

Fix $j \in\{1, \cdots, r\}$. For a point $v \in X_{j} \backslash\left(C_{\infty}\left(\Sigma_{X}\right) \cup C_{\infty}\left(\Sigma_{X}^{\prime}\right)\right)$ and for $\eta, R>0$ we define

$$
C_{\eta, R}\left(v^{\prime}\right):=\left\{w \in \mathbb{C}^{p} \mid \exists t>0 ;\left\|t v^{\prime}-w\right\| \leq \eta t\right\} \backslash B_{R}(0),
$$

where $v^{\prime}=\pi(v)$. Then, we consider a sufficiently small $\eta>0$ and larger $R>0$ such that $C_{\eta, R}\left(v^{\prime}\right) \subset \mathbb{C}^{p} \backslash \sigma(X) \cup \sigma\left(C_{\infty}(X)\right)$. Thus, the number of connected components of $\left(\left.\pi\right|_{X}\right)^{-1}\left(C_{\eta, R}\left(v^{\prime}\right)\right)$ (resp. $\left(\left.\pi\right|_{X_{j}}\right)^{-1}\left(C_{\eta, R}\left(v^{\prime}\right)\right)$ ) is equal to $\operatorname{deg}(X)$ (resp. $\left.\operatorname{deg}\left(X_{j}\right)\right)$. Moreover, there exist a connected component $V$ of $\left(\left.\pi\right|_{X_{j}}\right)^{-1}\left(C_{\eta, R}\left(v^{\prime}\right)\right)$ such that $v \in V$ and a compact subset $K \subset \mathbb{C}^{n}$ such that for each connected component $A_{i}$ of $\left(\left.\pi\right|_{X}\right)^{-1}\left(C_{\eta, R}\left(v^{\prime}\right)\right)$, we have $C_{\infty}\left(A_{i}\right) \cap\left(\mathbb{C}^{n} \backslash K\right) \subset\left(\left.\pi\right|_{C_{\infty}(X)}\right)^{-1}\left(C_{\eta, R}\left(v^{\prime}\right)\right)$. Then, we denote by $k_{X}^{\infty}(v)$ to be the number of connected components $A_{i}^{\prime}$ s such that $C_{\infty}\left(A_{i}\right) \cap\left(\mathbb{C}^{n} \backslash K\right) \subset V$. By definition, we can see that $k_{X}^{\infty}$ is locally constant and as $X_{j} \backslash\left(C_{\infty}\left(\Sigma_{X}\right) \cup C_{\infty}\left(\Sigma_{X}^{\prime}\right)\right)$ is connected, $k_{X}^{\infty}$ is constant on $X_{j} \backslash\left(C_{\infty}\left(\Sigma_{X}\right) \cup C_{\infty}\left(\Sigma_{X}^{\prime}\right)\right)$. Thus, we define $k_{X}^{\infty}\left(X_{j}\right)=k_{X}^{\infty}(v)$ and we call $k_{X}^{\infty}\left(X_{j}\right)$ the relative multiplicity at infinity of $X_{j}$ (over $X$ ). In particular, $k_{X}^{\infty}(w)=k_{X}^{\infty}(v)$ for all $w \in \pi^{-1}\left(v^{\prime}\right) \cap X_{j}$ and, therefore, we obtain

$$
\operatorname{deg}(X)=\sum_{j=0}^{r} k_{X}^{\infty}\left(X_{j}\right) \cdot \operatorname{deg}\left(X_{j}\right) .
$$

By taking $X=Y$ and $\varphi=$ id in the proof of Theorem 3.1. we see that the definition of $k_{X}^{\infty}\left(X_{j}\right)$ does not depend on $\pi$,

\section{Main results}

Let $Z \subset \mathbb{R}^{\ell}$ be a path connected subset. Given two points $q, \tilde{q} \in Z$, we recall that the inner distance in $Z$ between $q$ and $\tilde{q}$ is the number $d_{Z}(q, \tilde{q})$ below:

$$
d_{Z}(q, \tilde{q}):=\inf \{\operatorname{length}(\gamma) \mid \gamma \text { is an } \operatorname{arc} \text { on } Z \text { connecting } q \text { to } \tilde{q}\} .
$$

Theorem 3.1. Let $X \subset \mathbb{C}^{n}$ and $Y \subset \mathbb{C}^{m}$ be complex algebraic subsets, with pure dimension $p=\operatorname{dim} X=\operatorname{dim} Y$, and let $X_{1}, \ldots, X_{r}$ and $Y_{1}, \ldots, Y_{s}$ be the irreducible components of the tangent cones at infinity $C_{\infty}(X)$ and $C_{\infty}(Y)$ respectively. If $X$ and $Y$ are outer bi-Lipschitz homeomorphic at infinity, then $r=s$ and, up to a re-ordering of indices, $k_{X}^{\infty}\left(X_{j}\right)=k_{Y}^{\infty}\left(Y_{j}\right)$, $\forall j$. 
Proof. This proof shares its structure with the corresponding result in the local case in 5. By hypotheses there are compact subsets $K \subset \mathbb{C}^{n}$ and $\widetilde{K} \subset \mathbb{C}^{m}$ and an outer bi-Lipschitz homeomorphism $\varphi: X \backslash K \rightarrow Y \backslash \widetilde{K}$. Let $S=\left\{n_{k}\right\}_{k \in \mathbb{N}}$ be a sequence of positive real numbers such that

$$
n_{k} \rightarrow+\infty \quad \text { and } \quad \frac{\varphi\left(n_{k} v\right)}{n_{k}} \rightarrow d \varphi(v)
$$

where $d \varphi$ is the tangent map at infinity of $\varphi$ like in Theorem 2.3 (for more details, see 6], Theorem 4.5). Since, $d \varphi$ is an outer bi-Lipschitz homeomorphism, we get $r=s$ and there is a permutation $P:\{1, \ldots, r\} \rightarrow\{1, \ldots, s\}$ such that $d \varphi\left(X_{j}\right)=Y_{P(j)} \forall j$. This is why we can suppose $d \varphi\left(X_{j}\right)=Y_{j} \forall j$ up to a re-ordering of indices.

Let $\pi: \mathbb{C}^{n} \rightarrow \mathbb{C}^{p}$ and $\widetilde{\pi}: \mathbb{C}^{m} \rightarrow \mathbb{C}^{p}$ be linear projections such that

$$
\pi^{-1}(0) \cap C_{\infty}(X)=\{0\} \quad \text { and } \quad \tilde{\pi}^{-1}(0) \cap C_{\infty}(Y)=\{0\} .
$$

Let us denote the ramification locus of

$$
\left.\pi\right|_{X}: X \rightarrow \mathbb{C}^{p} \text { and }\left.\pi\right|_{C_{\infty}(X)}: C_{\infty}(X) \rightarrow \mathbb{C}^{p}
$$

by $\sigma(X)$ and $\sigma\left(C_{\infty}(X)\right)$ respectively. By similar way, we define $\sigma(Y)$ and $\sigma\left(C_{\infty}(Y)\right)$. Let us denote $\Sigma_{X}=\left(\left.\pi\right|_{X}\right)^{-1}(\sigma(X)), \Sigma_{X}^{\prime}=\left(\left.\pi\right|_{C_{\infty}(X)}\right)^{-1}\left(\sigma\left(C_{\infty}(X)\right)\right), \Sigma_{Y}=\left(\left.\widetilde{\pi}\right|_{Y}\right)^{-1}(\sigma(Y))$ and $\Sigma_{Y}^{\prime}=$ $\left(\left.\widetilde{\pi}\right|_{C_{\infty}(Y)}\right)^{-1}\left(\sigma\left(C_{\infty}(Y)\right)\right)$.

Let us suppose that there is $j \in\{1, \ldots, r\}$ such that $k_{X}^{\infty}\left(X_{j}\right)>k_{Y}^{\infty}\left(Y_{j}\right)$. Thus, given a unitary point $v \in X_{j} \backslash\left(C_{\infty}\left(\Sigma_{X}\right) \cup C_{\infty}\left(\Sigma_{X}^{\prime}\right)\right)$ such that $w=d \varphi(v) \in Y_{j} \backslash\left(C_{\infty}\left(\widetilde{\Sigma}_{Y}\right) \cup C_{\infty}\left(\widetilde{\Sigma}_{Y}^{\prime}\right)\right)$, let $\eta, R>0$ such that

$$
C_{\eta, R}\left(v^{\prime}\right) \subset \mathbb{C}^{p} \backslash\left(\sigma(X) \cup \sigma\left(C_{\infty}(X)\right)\right)
$$

and

$$
C_{\eta, R}\left(w^{\prime}\right) \subset \mathbb{C}^{p} \backslash\left(\sigma(Y) \cup \sigma\left(C_{\infty}(Y)\right)\right),
$$

where $v^{\prime}=\pi(v)$ and $w^{\prime}=\widetilde{\pi}(d \varphi(v))$. Therefore, there are at least two different connected components $V_{j i}$ and $V_{j l}$ of $\pi^{-1}\left(C_{\eta, R}\left(v^{\prime}\right)\right) \cap X$ and sequences $\left\{z_{k}\right\}_{k \in \mathbb{N}} \subset V_{j i}$ and $\left\{w_{k}\right\}_{k \in \mathbb{N}} \subset V_{j l}$ such that $t_{k}=\left\|z_{k}\right\|=\left\|w_{k}\right\| \in S=\left\{n_{k}\right\}_{k \in \mathbb{N}}, \lim \frac{1}{t_{k}} z_{k}=\lim \frac{1}{t_{k}} w_{k}=v$ and $\varphi\left(z_{k}\right), \varphi\left(w_{k}\right) \in \widetilde{V}_{j m}$, where $\widetilde{V}_{j m}$ is a connected component of $\widetilde{\pi}^{-1}\left(C_{\eta, R}\left(w^{\prime}\right)\right) \cap Y$.

Let us choose linear coordinates $(x, y)$ in $\mathbb{C}^{m}$ such that $\widetilde{\pi}(x, y)=x$.

Claim. There exist a compact subset $K \subset \mathbb{C}^{m}$ and a constant $C>0$ such that $\|y\| \leq C\|x\|$ for all $(x, y) \in Y \backslash K$.

If this Claim is not true, there exists a sequence $\left\{\left(x_{k}, y_{k}\right)\right\} \subset Y$ such that $\lim _{k \rightarrow+\infty}\left\|\left(x_{k}, y_{k}\right)\right\|=$ $+\infty$ and $\left\|y_{k}\right\|>k\left\|x_{k}\right\|$. Up to subsequence, one can suppose that $\lim _{k \rightarrow+\infty} \frac{y_{k}}{\left\|y_{k}\right\|}=y_{0}$. Since $\frac{\left\|x_{k}\right\|}{\left\|y_{k}\right\|}<$ $\frac{1}{k},\left(0, y_{0}\right) \in C_{\infty}(Y)$, which is a contradiction, because $y_{0} \neq 0,\left(0, y_{0}\right) \in \pi^{-1}(0)$ and $\widetilde{\pi}^{-1}(0) \cap$ $C_{\infty}(Y)=\{0\}$. Therefore, the Claim is true. In particular, $V=\widetilde{V}_{j m}$ is outer bi-Lipschitz homeomorphic to $C_{\eta, R}\left(w^{\prime}\right)$ and since $\varphi\left(z_{k}\right), \varphi\left(w_{k}\right) \in \widetilde{V}_{j m} \forall k \in \mathbb{N}$, we have

$$
\left\|\varphi\left(z_{k}\right)-\varphi\left(w_{k}\right)\right\|=o_{\infty}\left(t_{k}\right)
$$

and

$$
d_{Y}\left(\varphi\left(z_{k}\right), \varphi\left(w_{k}\right)\right) \leq d_{V}\left(\varphi\left(z_{k}\right), \varphi\left(w_{k}\right)\right)=o_{\infty}\left(t_{k}\right),
$$

where $g\left(t_{k}\right)=o_{\infty}\left(t_{k}\right)$ means $\lim _{k \rightarrow \infty} \frac{g\left(t_{k}\right)}{t_{k}}=0$. Now, since $X$ is outer bi-Lipschitz homeomorphic to $Y$, we have $d_{X}\left(z_{k}, w_{k}\right) \leq o_{\infty}\left(t_{k}\right)$. On the other hand, since $z_{k}$ and $w_{k}$ lie in different connected components of $\pi^{-1}\left(C_{\eta, R}\left(v^{\prime}\right)\right) \cap X$, there exists a constant $C>0$ such that $d_{X}\left(z_{k}, w_{k}\right) \geq C t_{k}$, which is a contradiction.

We have proved that $k_{X}^{\infty}\left(X_{j}\right) \leq k_{Y}^{\infty}\left(Y_{j}\right), j=1, \cdots, r$. By the same arguments, using that $\varphi^{-1}$ is an outer bi-Lipschitz map, we also obtain $k_{Y}^{\infty}\left(Y_{j}\right) \leq k_{X}^{\infty}\left(X_{j}\right), j=1, \cdots, r$. 


\subsection{Degree as an outer bi-Lipschitz invariant at infinity}

The first application of our main results proved in the previous section is the outer biLipschitz invariance of the degree of complex algebraic curves in $\mathbb{C}^{n}$.

Corollary 3.2. Let $X \subset \mathbb{C}^{n}$ and $Y \subset \mathbb{C}^{m}$ be complex algebraic subsets, with $\operatorname{dim} X=$ $\operatorname{dim} Y=1$. If $X$ and $Y$ are outer bi-Lipschitz homeomorphic at infinity, then $\operatorname{deg}(X)=\operatorname{deg}(Y)$.

Proof. Let $X_{1}, \ldots, X_{r}$ and $Y_{1}, \ldots, Y_{s}$ be the irreducible components of the tangent cones at infinity $C_{\infty}(X)$ and $C_{\infty}(Y)$ respectively. Since $\operatorname{dim} X=\operatorname{dim} Y=1$, we have that $X_{1}, \ldots, X_{r}$ and $Y_{1}, \ldots, Y_{s}$ are complex lines. Thus,

$$
\operatorname{deg}\left(X_{1}\right)=\cdots=\operatorname{deg}\left(X_{r}\right)=\operatorname{deg}\left(Y_{1}\right)=\cdots=\operatorname{deg}\left(Y_{s}\right)=1
$$

and using Equality 2.1. we get $\operatorname{deg}(X)=\sum_{j=0}^{r} k_{X}^{\infty}\left(X_{j}\right)$ and $\operatorname{deg}(Y)=\sum_{j=0}^{s} k_{X}^{\infty}\left(Y_{j}\right)$. Therefore, by Theorem 3.1, $\operatorname{deg}(\bar{X})=\operatorname{deg}(Y)$.

Let us fix $d \in \mathbb{N}$.

THEOREM 3.3. The statements below are equivalent.

$\tilde{A} 1(d)$ Let $X \subset \mathbb{C}^{n}$ and $Y \subset \mathbb{C}^{m}$ be two complex analytic sets with $\operatorname{dim} X=\operatorname{dim} Y=d$. If their germs at $0 \in \mathbb{C}^{n}$ and $0 \in \mathbb{C}^{m}$, respectively, are outer bi-Lipschitz homeomorphic, then $m(X, 0)=m(Y, 0)$.

$A 1(d)$ Let $X \subset \mathbb{C}^{n}$ and $Y \subset \mathbb{C}^{m}$ be two complex algebraic sets with $\operatorname{dim} X=\operatorname{dim} Y=d$. If $X$ and $Y$ are outer bi-Lipschitz homeomorphic at infinity, then $\operatorname{deg}(X)=\operatorname{deg}(Y)$.

\section{Proof.}

As we pointed out in the introduction of the paper; we know from 5 that statement $\tilde{A} 1(d)$ holds true if and only if it is true by considering just homogeneous complex algebraic sets. But, if $A \subset \mathbb{C}^{n}$ is a homogeneous complex algebraic set, then $\operatorname{deg}(A)=m(A, 0)$.

From now, we are ready to start the proof of the theorem. First, let us suppose that statement $\mathrm{A} 1(d)$ is true. Since cones which are outer bi-Lipschitz homeomorphic as germs at their vertices are globally outer bi-Lipschitz homeomorphic, as was remarked in [13, it follows from the above observation that $\tilde{\mathrm{A}} 1(d)$ holds true as well. Secondly, let us suppose that $\tilde{\mathrm{A}} 1(d)$ holds true. Let $X \subset \mathbb{C}^{n}$ and $Y \subset \mathbb{C}^{m}$ be two complex algebraic sets with $d=\operatorname{dim} X=\operatorname{dim} Y$. Let us suppose that $X$ and $Y$ are outer bi-Lipschitz homeomorphic at infinity. Then, there exist $K \subset \mathbb{C}^{n}$ and $\widetilde{K} \subset \mathbb{C}^{m}$ two compact subsets and a outer bi-Lipschitz homeomorphism $\varphi: X \backslash K \rightarrow Y \backslash \widetilde{K}$. Let us denote by $X_{1}, \ldots, X_{r}$ and $Y_{1}, \ldots, Y_{s}$ the irreducible components of the cones $C_{\infty}(X)$ and $C_{\infty}(Y)$ respectively. It comes from Theorem 3.1 that $r=s$ and the outer bi-Lipschitz homeomorphism $d \varphi: C_{\infty}(X) \rightarrow C_{\infty}(Y)$, up to re-ordering of indices, sends $X_{i}$ onto $Y_{i}$ and $k_{X}^{\infty}\left(X_{i}\right)=k_{Y}^{\infty}\left(Y_{i}\right) \forall i$. Furthermore, $d \varphi(0)=0$.

By Proposition 2.2. the tangent cones at infinity $C_{\infty}(X)$ and $C_{\infty}(Y)$ are homogeneous complex algebraic subsets. Thus, the irreducible components $X_{1}, \ldots, X_{r}$ and $Y_{1}, \ldots, Y_{s}$ are homogeneous complex algebraic subsets as well. Since $\tilde{A} 1(d)$ is true, we have $m\left(X_{i}, 0\right)=$ $m\left(Y_{i}, 0\right) \forall i$, hence $\operatorname{deg}\left(X_{i}\right)=\operatorname{deg}\left(Y_{i}\right) \forall i$. Finally, by using Equality 2.1. we get $\operatorname{deg}(X)=$ $\operatorname{deg}(Y)$ which give us that $\mathrm{A} 1(d)$ is true. 
(1) Let $X \subset \mathbb{C}^{N+1}$ and $Y \subset \mathbb{C}^{M+1}$ be two complex analytic surfaces. If $(X, 0)$ and $(Y, 0)$ are outer bi-Lipschitz homeomorphic, then $m(X, 0)=m(Y, 0)$.

(2) Let $X \subset \mathbb{C}^{N+1}$ and $Y \subset \mathbb{C}^{M+1}$ be two complex algebraic surfaces. If $X$ and $Y$ are outer bi-Lipschitz homeomorphic at infinity, then $\operatorname{deg}(X)=\operatorname{deg}(Y)$.

Proof. By Theorem 2.1 in [5], it is enough to show (1) when $X$ and $Y$ are two irreducible homogeneous complex algebraic sets. The proof of Theorem 2.1 in [5] shows that (1) is equivalent to the following statement: let $(S, 0)$ and $\left(S^{\prime}, 0\right)$ be homogeneous irreducible affine algebraic surfaces. If $S \backslash\{0\}$ is bi-lipschitz homeomorphic to $S^{\prime} \backslash\{0\}$, then we have the equality $\operatorname{deg}(S)=\operatorname{deg}\left(S^{\prime}\right)$. Indeed, the reader may review the details of the proof of Theorem 2.1 in 5 and check that ambient bi-lipschitz homeomorphism can be replaced by outer-bilipschitz homeomorphism in the statement of the theorem.

Thus, the part (1) of the theorem is reduced to prove the following proposition.

Proposition 3.5. Let $(S, 0)$ and $\left(S^{\prime}, 0\right)$ be homogeneous irreducible affine algebraic surfaces. If $S \backslash\{0\}$ is homeomorphic to $S^{\prime} \backslash\{0\}$, then we have the equality $\operatorname{deg}(S)=\operatorname{deg}\left(S^{\prime}\right)$.

Proof. We have the isomorphism $H^{2}(S \backslash\{0\} ; \mathbb{Z}) \cong H^{2}\left(S^{\prime} \backslash\{0\} ; \mathbb{Z}\right)$. Hence it is enough to show that the torsion part of this cohomology group is isomorphic to $\mathbb{Z} / \operatorname{deg}(S) \mathbb{Z}$.

Let $\pi: S \backslash\{0\} \rightarrow \mathbb{P}(S)$ denote the quotient map by the $\mathbb{C}^{*}$-action. The fibration $\pi$ is a pullback of the tautological bundle minus the zero section over the projective space $\mathbb{P}^{N}$ where $\mathbb{P}(S)$ is embedded. Hence the higher direct images $R^{q} \pi_{*} \underline{\mathbb{Z}}_{S \backslash\{0\}}$ form local systems whose stalk is the $q$-th cohomology group of the fibre of the fibration $\pi$. Since the fibre of $\pi$ is $\mathbb{C}^{*}$ we have the vanishing $R^{q} \pi_{*} \underline{\mathbb{Z}}_{S \backslash\{0\}}$ for $q \neq 0,1$. Morever $R^{q} \pi_{*} \underline{\mathbb{Z}}_{S \backslash\{0\}}$ is the pullback from the corresponding local systems over $\mathbb{P}^{N}$. Since the projective space is simply-connected the local systems have trivial monodromy. Hence, the second page of the Leray spectral sequence for $\pi$ (see $[\mathbf{1 5}, 5.8 .6$, page 152) is

$$
E_{2}^{p, q}=H^{p}(\mathbb{P}(S), \mathbb{Z}) .
$$

Since $E_{2}^{p, q}$ vanishes for $p \neq 0,1,2$ and $q \neq 0,1$, the differentials $d_{i}$ are all zero for $i \geq 3$ and the only non-zero $d_{2}$ differential is:

$$
d_{2}: H^{0}(\mathbb{P}(S), \mathbb{Z}) \cong \mathbb{Z} \rightarrow H^{2}(\mathbb{P}(S), \mathbb{Z}) \cong \mathbb{Z}
$$

which coincides with multiplication by the first Chern class of the tautological bundle over $\mathbb{P}^{N}$. Hence $d_{2}$ is multiplication by $\operatorname{deg}(S)$. We deduce the isomorphisms

$$
\begin{gathered}
E_{\infty}^{0,2}=E_{2}^{0,2}=0, \\
E_{\infty}^{1,1} \cong E_{2}^{1,1} \cong \mathbb{Z}^{b_{1}}, \\
E_{\infty}^{2,0} \cong E_{3}^{2,0} \cong \mathbb{Z} / \operatorname{deg}(S) \mathbb{Z},
\end{gathered}
$$

where $b_{1}$ is the first Betti number of $\mathbb{P}(S)$.

By the vanishing of $E_{\infty}^{0,2}$ there is a short exact sequence

$$
0 \rightarrow E_{\infty}^{2,0} \rightarrow H^{2}(S \backslash\{0\}, \mathbb{Z}) \rightarrow E_{\infty}^{1,1} \rightarrow 0,
$$

which splits by the freeness of $E_{\infty}^{1,1}$. Hence the torsion part of $H^{2}(S \backslash\{0\}, \mathbb{Z})$ is isomorphic to $\mathbb{Z} / \operatorname{deg}(S) \mathbb{Z}$ as needed. 
Now we prove part (2) of the main theorem. Since we have proved part (1), we see that the statement $\tilde{A} 1(2)$ of Theorem 3.3 has a positive answer, hence $A 1(2)$ has a positive answer as well. Therefore, part (2) is proved.

Let us denote by $\mathcal{C}_{1, \infty}$ the set of all complex algebraic sets $X \subset \mathbb{C}^{n}$, such that each irreducible component $X_{j}$ of $C_{\infty}(X)$ satisfies $\operatorname{dim} \operatorname{Sing}\left(X_{j}\right) \leq 1$.

Theorem 3.6. Let $f, g: \mathbb{C}^{n} \rightarrow \mathbb{C}$ be two polynomials. Suppose that $V(f) \in \mathcal{C}_{1, \infty}$. Suppose there exist compact subsets $K, \widetilde{K} \subset \mathbb{C}^{n}$ and an outer bi-Lipschitz homeomorphism $\varphi: \mathbb{C}^{n} \backslash K \rightarrow \mathbb{C}^{n} \backslash \widetilde{K}$ such that $\varphi(V(f) \backslash K)=V(g) \backslash \widetilde{K}$. Then $V(g) \in \mathcal{C}_{1, \infty}$ and $\operatorname{deg}(V(f))=$ $\operatorname{deg}(V(g))$.

Proof. By the proof of Theorem 3.1, we can suppose that $f$ and $g$ are irreducible homogeneous polynomials. By Theorem 5.4 in $\mathbf{1 4}$ and using the same arguments as in the very beginning of the proof of Theorem 3.3, it follows that $\operatorname{deg}(V(f))=\operatorname{deg}(V(g))$.

\section{References}

1. L. Birbrair, A. Fernandes, J. E. Sampaio and M. Verbitsky, Multiplicity of singularities is not a bi-Lipschitz invariant. Preprint arXiv:1801.06849v1 [math.AG], (2018).

2. E. M. Chirka, Complex analytic sets. Kluwer Academic Publishers, 1989.

3. G. Comte, Multiplicity of complex analytic sets and bi-Lipschitz maps. Real analytic and algebraic singularities (Nagoya/Sapporo/Hachioji, 1996) Pitman Res. Notes Math. Ser., 381 (1998), 182-188.

4. A. Fernandes, Topological equivalence of complex curves and bi-Lipschitz maps. Michigan Math. J., 51 (2003), 593-606.

5. A. Fernandes and J. E. Sampaio, Multiplicity of analytic hypersurface singularities under bi-Lipschitz homeomorphisms. Journal of Topology, 9 (2016), 927-933.

6. A. Fernandes and J. E. Sampaio, On Lipschitz rigidity of complex analytic sets. Preprint arXiv:1705.03085v3 [math.AG], (2018).

7. K. Kurdyka and G. Raby, Densité des ensembles sous-analytiques. Ann. Inst. Fourier (Grenoble), 39 (1989), n. 3, 753-771.

8. C.-T. LÊ and T.-S. PhAm, On tangent cones at infinity of algebraic varieties. Journal of Algebra and Its Applications, 16 (2018), n. 2, 1850143 (10 pages).

9. A. Nemethi, Five lectures on normal surface singularities Lectures delivered at the Summer School in "Low dimensional topology", Budapest, Hungary, 1998; Proceedings of the Summer School, Bolyai Society Mathematical Studies, 8, Low Dimensional Topology, (1999) 269-351.

10. W. Neunmann and A. Pichon, Lipschitz geometry of complex curves. Journal of Singularities, 10 (2014), $225-234$.

11. W. Neunmann and A. Pichon, Lipschitz geometry of complex surfaces: analytic invariants and equisingularity. Preprint arXiv:1211.4897v3 [math.AG], (2016).

12. F. Pham and B. Teissier, Fractions lipschitziennes d'une algèbre analytique complexe et saturation de Zariski. Prépublications du Centre de Mathématiques de l'Ecole Polytechnique (Paris), No. M17.0669, June (1969). Available at https://hal.archives-ouvertes.fr/hal-00384928/

13. J. E. SAmpaio, Bi-Lipschitz homeomorphic subanalytic sets have bi-Lipschitz homeomorphic tangent cones. Selecta Math. (N.S.), 22 (2016), n. 2, 553-559.

14. J. E. Sampaio, Multiplicity, regularity and blow-spherical equivalence of complex analytic sets. Preprint arXiv:1702.06213v2 [math.AG], (2017).

15. C. A. Weibel, An introduction to homological algebra. Cambridge University Press, 1994.

16. O. Zariski, Some open questions in the theory of singularities. Bull. of the Amer. Math. Soc., 77 (1971), n. $4,481-491$. 
Javier Fernández de Bobadilla

(1) IKERBASQUE, Basque Foundation for Science, Maria Diaz de Haro 3, 48013, Bilbao, Bizkaia, Spain.

(2) BCAM Basque Center for Applied Mathematics, Mazarredo 14, E48009 Bilbao, Basque Country, Spain.

jbobadilla@bcamath.org

J. Edson Sampaio

(1) Departamento de Matemática, Universidade Federal do Ceará, Rua Campus do Pici, s/n, Bloco 914, Pici, 60440-900, Fortaleza-CE, Brazil.

(2) BCAM - Basque Center for Applied Mathematics, Mazarredo, 14 E48009 Bilbao, Basque Country - Spain.

edsonsampaio@mat.ufc.br esampaio@bcamath.org
Alexandre Fernandes

Departamento de Matemática, Universidade Federal do Ceará, Rua Campus do Pici, s/n, Bloco 914, Pici, 60440-900, Fortaleza-CE Brazil.

alex@mat.ufc.br 